\title{
A Variational Iteration Method Involving Adomian Polynomials for a Strongly Nonlinear Boundary Value Problem
}

\author{
Shih-Hsiang Chang*
}

Department of Mechanical Engineering, Far East University, Tainan 74448, Taiwan, R.O.C.

Received 4 November 2016; Accepted (in revised version) 29 November 2018.

\begin{abstract}
A variational iteration method involving Adomian polynomials to solve a strongly nonlinear boundary value problem is considered. After its convergence is established, the efficiency and accuracy of the proposed method are tested on problems with exponential nonlinearity.
\end{abstract}

AMS subject classifications: 34B16, 65G99, 65K10, 65L20, 68U20

Key words: Boundary value problem, variational iteration method, Adomian polynomials, convergence.

\section{Introduction}

The nonlinear boundary value problem

$$
\begin{aligned}
& y^{\prime \prime}+\frac{m}{x} y^{\prime}+f(x, y)=0, \quad 0 \leq x \leq 1, \\
& \alpha_{1} y(0)+\beta_{1} y^{\prime}(0)=\gamma_{1}, \quad m=0 \quad \text { or } y^{\prime}(0)=0, \quad m>0, \\
& \alpha_{2} y(1)+\beta_{2} y^{\prime}(1)=\gamma_{2},
\end{aligned}
$$

where $\alpha_{i}, \beta_{i}, \gamma_{i}, i=1,2$ are finite constants, arises in various applications, including thermal explosions [10], tumor growth models [7], electroosmotic flows [11,12,15], modelling of heat sources in human head [26], and oxygen diffusion [42]. The unique solvability of the problem (1.1) for $m \geq 1$ and boundary conditions $y^{\prime}(0)=0$ and $y(1)=B$ was established by Chawla \& Shivkumar [19], while the more general case of nonlinear boundary conditions was studied by Garner \& Shivaji [27]. In order to find approximate solutions of the problem, various numerical methods have been used - e.g. the Adomian decomposition method [39], the Taylor series method [18], a variational iteration method [49, 58]. The first two of these methods experience convergence difficulties, while the third one is

*Corresponding author. Email addresses: shchang@mail.feu.edu.tw (S.-H. Chang) 
restricted to the solution of the problem (1.1) with functions $f(x, y)$ belonging to a special class of non-linear polynomials. To avoid these difficulties, modifications of the last two methods have been suggested $[13,14,16]$.

The variational iteration method proposed by $\mathrm{He}[30-32]$ and its modifications $[38,56]$ have a high convergence rate and small error, so they are widely regarded as a good tool for solving functional equations $[1,28,29,33,34,37,41,44,45,51,53,59]$ arising in nonlinear science and engineering problems $[5,6,40,50,54,55,60,61]$. Based on the variational iteration algorithm I, the convergence of the method has been extensively studied in [29, 45,51-53]. Recently, Chang [17] used the variational iteration algorithm II to prove the convergence of the method for two-point diffusion problems. For more details about the method and its applications we refer the reader to [36,37] and references therein.

The concept of Adomian polynomials was introduced by Adomian [8] in 1976. Later, Adomian and Rach [9] presented a formal formula to generate the Adomian polynomials for all form of nonlinearity. Since then, various algorithms for calculating the Adomian polynomials have been proposed to improve computational efficiency $[2,22,23,47,57]$. Symbolic implementation of several recurrence algorithms by using MATHEMATICA or MAPLE was also developed - cf. $[20,21,23,46]$. The convergence of the Adomian polynomial series has been also discussed in $[2,22,48]$.

Here, we combine a variational iteration method with Adomian polynomials to obtain the approximate solutions of a strongly nonlinear boundary value problem. In contrast to the above mentioned methods, our approach does not require any additional tools. The key idea is that the nonlinear terms in the correction functionals are decomposed into a series of Adomian polynomials, as this simplifies the computations considerably. Sufficient conditions for the method convergence are established, and test examples involving exponential nonlinearity, demonstrate the efficiency of the algorithm. The errors do not depend on a specific location of a point $x \in[0,1]$, and are valid for the whole domain considered. This approach can be applied to various problems involving other differential equations with a strong nonlinearity.

\section{Convergence}

According to He [35, 36] and Chang [17], the variational iteration algorithms I and II for the problem (1.1) have the form

$$
\begin{aligned}
& y_{n+1}(x)=y_{n}(x)+\int_{0}^{x} \lambda(s ; x)\left[y_{n}^{\prime \prime}(s)+\frac{m}{s} y_{n}^{\prime}(s)+f\left(s, y_{n}(s)\right)\right] \mathrm{d} s, \quad n \geq 0, \\
& y_{n+1}(x)=y_{0}(x)+\int_{0}^{x} \lambda(s ; x) f\left(s, y_{n}(s)\right) \mathrm{d} s, \quad n \geq 0
\end{aligned}
$$

where $y_{0}(x):=y(0)+y^{\prime}(0) x$ and $\lambda(s ; x)$ is the Lagrange multiplier $[49,58]$ defined by 


$$
\lambda(s ; x)=\left\{\begin{array}{l}
s \ln \left(\frac{s}{x}\right), \quad m=1, \\
\frac{s\left(s^{m-1}-x^{m-1}\right)}{(m-1) x^{m-1}}, \quad 0 \leq m \neq 1 .
\end{array}\right.
$$

Integrating the differential equation in (1.1) twice, one obtains

$$
\begin{aligned}
& y^{\prime}(x)=\left\{\begin{array}{l}
y^{\prime}(0)-\int_{0}^{x} f(s, y(s)) \mathrm{d} s, \quad m=0, \\
-\int_{0}^{x} \frac{s^{m}}{x^{m}} f(s, y(s)) \mathrm{d} s, \quad m>0,
\end{array}\right. \\
& y(x)=y_{0}(x)+\int_{0}^{x} \lambda(s ; x) f(s, y(s)) \mathrm{d} s .
\end{aligned}
$$

Though the variational iteration method is widely used in nonlinear problems, the presence of complicated functions in the kernels of symbolic integrals can cause substantial difficulties in implementation. Therefore, we represent the nonlinear term $f(x, y(x))$ as the series

$$
f(x, y(x))=\sum_{k=0}^{\infty} A_{k}\left(u_{0}(x), u_{1}(x), \cdots, u_{k}(x)\right),
$$

where $u_{0}(x)=y_{0}(x), u_{k}(x)=y_{k}(x)-y_{k-1}(x), A_{0}\left(u_{0}(x)\right)=f\left(x, u_{0}(x)\right)$, and for $k \geq 1$ the Adomian polynomials $A_{k}(x)$ are recurrently defined in [23] by

$$
A_{k}(x)=A_{k}\left(u_{0}(x), u_{1}(x), \cdots, u_{k}(x)\right)=\sum_{i=1}^{k} f^{(i)}\left(x, u_{0}(x)\right) C_{k}^{i}(x), \quad k \geq 1
$$

with

$$
C_{k}^{1}(x):=u_{k}(x), \quad k \geq 1, \quad C_{k}^{i}(x):=\frac{1}{k} \sum_{j=0}^{k-i}(j+1) u_{j+1}(x) C_{k-1-j}^{i-1}(x), \quad 2 \leqslant i \leqslant k .
$$

Such a modification is called the variational iteration-Adomian method $[3,4,24,25]$. Analogously, taking into account the Eq. (2.4), one obtains

$$
y(x)=y_{0}(x)+\int_{0}^{x} \lambda(s ; x) \sum_{k=0}^{\infty} A_{k}(s) \mathrm{d} s
$$

and for $n \geq 0$ the variational iteration algorithm I and algorithm II take the form

$$
\begin{aligned}
& y_{n+1}(x)=y_{n}(x)+\int_{0}^{x} \lambda(s ; x)\left[y_{n}^{\prime \prime}(s)+\frac{m}{s} y_{n}^{\prime}(s)+\sum_{k=0}^{n} A_{k}(s)\right] \mathrm{d} s, \\
& y_{n+1}(x)=y_{0}(x)+\int_{0}^{x} \lambda(s ; x) \sum_{k=0}^{n} A_{k}(s) \mathrm{d} s .
\end{aligned}
$$


The Adomian polynomials $A_{k}(x)$ above are also called the classical Adomian polynomials. According to [48], they satisfy the relations

$$
\begin{aligned}
& A_{0}\left(u_{0}(x)\right)=f\left(x, u_{0}(x)\right)=f\left(x, y_{0}(x)\right), \\
& A_{k}(x)<f\left(x, y_{k}(x)\right)-f\left(x, y_{k-1}(x)\right), \quad k \geq 1 .
\end{aligned}
$$

Following Ref. [17], we present the truncation error for the method under consideration and establish sufficient conditions for its convergence.

Theorem 2.1. Assume that the function $f(x, y)$ is analytic in the rectangle $R=\{(x, y): 0 \leq$ $\left.x \leq 1,\left|y-y_{0}\right| \leq b\right\}$ and bounded from above by an $M$ such that $M<2(m+1) b$. Then every sequence (2.8) or (2.9) with $y_{0}(x)=y(0)+y^{\prime}(0) x$ converges to the exact solution $y(x)$ of the problem (1.1) in the norm of $C_{[0,1]}$. Moreover,

$$
E_{n}:=\left\|y(x)-y_{n}(x)\right\|_{C_{[0,1]}} \leq \frac{M K^{n}}{(n+1) ! 2^{n+1}(m+1)(m+3) \cdots(m+2 n+1)},
$$

where $K=\|\partial f(x, y) / \partial y\|_{C_{[0,1]}}$.

Proof. Since $f(x, y)$ is analytic in $R$, by the Cauchy-Kovalevskaya theorem the problem (1.1) has unique solution $y(x)$ analytic in $x \in[0,1]$. Therefore, the Murray-Miller theorem for existence and uniqueness [43] guarantees that $f(x, y)=\sum_{k=0}^{\infty} A_{k}\left(u_{0}, u_{1}, \cdots, u_{k}\right)$, where $A_{k}(x)$ is the classical Adomian polynomials, and the above series is uniformly convergent because it is essentially a rearrangement of the parameterised Taylor expansion series of the analytic function $f(x, y)$ about the function $u_{0}(x)$ [48]. Obviously, $\left(x, y_{0}\right) \in R$ for all $x \in[0,1]$ such that $\left|f\left(x, y_{0}\right)\right|=\left|A_{0}(x)\right| \leq M$. It follows from (2.9) and (A.4) that for $n=0$ one has

$$
\left|y_{1}(x)-y_{0}(x)\right| \leq \int_{0}^{x}|\lambda(s ; x)|\left|A_{0}(s)\right| \mathrm{d} s \leq \frac{M}{2(m+1)} x^{2} \leq \frac{M}{2(m+1)}<b,
$$

so that $\left(x, y_{1}\right) \in R$ and $\left|f\left(x, y_{1}\right)\right| \leq M$. Further, the usual induction arguments show that $\left(x, y_{n}\right) \in R$ and $\left|f\left(x, y_{n}\right)\right| \leq M$ for any positive integer $n$. Thus the expressions containing the points $y_{n}(x), n \in \mathbb{N}$ can be estimated - e.g. the Eqs. (2.7) and (A.4) yield

$$
\begin{aligned}
\left|y(x)-y_{0}(x)\right| & \leq \int_{0}^{x}|\lambda(s ; x)|\left|\sum_{k=0}^{\infty} A_{k}\left(u_{0}, u_{1}, \cdots, u_{k}\right)\right| \mathrm{d} s=\int_{0}^{x}|\lambda(s ; x)||f(s, y(s))| \mathrm{d} s \\
& \leq M \int_{0}^{x}|\lambda(s ; x)| \mathrm{d} s \leq \frac{M}{2(m+1)} x^{2} \leq \frac{M}{2(m+1)} .
\end{aligned}
$$

Since $f(x, y)$ is an analytic function in $R$ and $\left(x, y_{n}\right) \in R$, there is a constant $K=$ $\|\partial f(x, y) / \partial y\|_{C_{[0,1]}}$ such that

$$
\left|f(x, y)-f\left(x, y_{n}\right)\right| \leq K\left|y(x)-y_{n}(x)\right|, \quad x \in[0,1] .
$$


The Eq. (2.10) implies that

$$
\left|\sum_{k=n+1}^{\infty} A_{k}\left(u_{0}, u_{1}, \cdots, u_{k}\right)\right| \leq K\left|y(x)-y_{n}(x)\right|, \quad x \in[0,1] .
$$

Analogously, if $x \in[0,1]$, then

$$
\begin{aligned}
\left|y(x)-y_{1}(x)\right| & \leq \int_{0}^{x}|\lambda(s ; x)|\left|\sum_{k=1}^{\infty} A_{k}\left(u_{0}, u_{1}, \cdots, u_{k}\right)\right| \mathrm{d} s \\
& \leq K \int_{0}^{x}|\lambda(s ; x)|\left|y(s)-y_{0}(s)\right| \mathrm{d} s \\
& \leq \frac{M K}{2(m+1)} \int_{0}^{x}\left|\lambda(s, x) s^{2}\right| \mathrm{d} s \leq \frac{M K}{2 ! 2^{2}(m+1)(m+3)} x^{4} \\
& \leq \frac{M K}{2 ! 2^{2}(m+1)(m+3)} .
\end{aligned}
$$

Continuing in this way, we eventually arrive at the estimate

$$
\left|y(x)-y_{n}(x)\right| \leq \frac{M K^{n}}{(n+1) ! 2^{n+1}(m+1)(m+3) \cdots(m+2 n+1)}
$$

valid for all $x \in[0,1]$. Therefore, the inequality (2.11) holds and $E_{n} \rightarrow 0$ as $n \rightarrow \infty$.

\section{Applications}

In this section, we consider approximate solution of several boundary value problems (1.1) found with the assistance of MATHEMATICA 5.2 on a laptop with a Pentium M $1.4 \mathrm{GHz}$ and $256 \mathrm{MB}$ of RAM.

Example 3.1. The nonlinear singular boundary value problem

$$
\begin{aligned}
& y^{\prime \prime}+\frac{1}{x} y^{\prime}+\mathrm{e}^{y}=0, \\
& y^{\prime}(0)=0, \quad y(1)=0
\end{aligned}
$$

arises in the theory of thermal explosions [10].

For $m=1$, the correction functional has the form

$$
y_{n+1}(x)=y_{n}(x)+\int_{x_{0}}^{x} s \ln \left(\frac{s}{x}\right)\left[y_{n}^{\prime \prime}(s)+\frac{1}{s} y_{n}^{\prime}(s)+\mathrm{e}^{y(s)}\right] \mathrm{d} s .
$$

Choosing a constant $a$ as the initial approximation $y_{0}$, we obtain

$$
y_{1}(x)=a-\frac{\mathrm{e}^{a}}{4} x^{2}
$$


However, the second and further consecutive iterations cannot be computed in a closed form due to complicated integrands. This is a common complication in the implementation of the variational iteration method for strongly nonlinear problems. In contrast, in the variational iteration-Adomian method, the term $y_{n+1}(x)$ is calculated by the formula

$$
y_{n+1}(x)=y_{n}(x)+\int_{0}^{x} s \ln \left(\frac{s}{x}\right)\left[y_{n}^{\prime \prime}(s)+\frac{1}{s} y_{n}^{\prime}(s)+\sum_{k=0}^{n} A_{k}(s)\right] \mathrm{d} s .
$$

According to the algorithm (2.6), the first four Adomian polynomials are

$$
\begin{aligned}
& A_{0}=\mathrm{e}^{u_{0}}, \quad A_{1}=\mathrm{e}^{u_{0}} u_{1}, \\
& A_{2}=\frac{1}{2} \mathrm{e}^{u_{0}} u_{1}^{2}+\mathrm{e}^{u_{0}} u_{2}, \\
& A_{3}=\frac{1}{6} \mathrm{e}^{u_{0}} u_{1}^{3}+\mathrm{e}^{u_{0}} u_{1} u_{2}+\mathrm{e}^{u_{0}} u_{3}
\end{aligned}
$$

produce the following approximations of the solution $y$ :

$$
\begin{aligned}
& y_{1}(x)=a-\frac{\mathrm{e}^{a}}{4} x^{2}, \\
& y_{2}(x)=a-\frac{\mathrm{e}^{a}}{4} x^{2}+\frac{\mathrm{e}^{2 a}}{64} x^{4}, \\
& y_{3}(x)=a-\frac{\mathrm{e}^{a}}{4} x^{2}+\frac{\mathrm{e}^{2 a}}{64} x^{4}-\frac{\mathrm{e}^{3 a}}{768} x^{6}, \\
& y_{4}(x)=a-\frac{\mathrm{e}^{a}}{4} x^{2}+\frac{\mathrm{e}^{2 a}}{64} x^{4}-\frac{\mathrm{e}^{3 a}}{768} x^{6}-\frac{\mathrm{e}^{4 a}}{8192} x^{8} .
\end{aligned}
$$

It follows from the Eq. (2.3) that $y^{\prime}(x) \leq 0$ for all $x \in[0,1]$. Hence, $y(x)$ is a nonincreasing function $(0 \leq y(x) \leq a$ for $0 \leq x \leq 1)$, which implies $M=K=\mathrm{e}^{a}$. Thus the sequence $y_{n}(x)$ converges to the exact solution of the problem (3.1) in the space $C_{[0,1]}$ and

$$
E_{n} \leq \frac{\mathrm{e}^{a(n+1)}}{2^{2(n+1)}(n+1) !(n+1) !}
$$

The unknown constant $a$ is to be determined by imposing a boundary condition on $y_{n}$ at $x=1$ to obtain a transcendental equation, $y_{n}(1)=0$. MATHEMATICA has a built-in command to solve this transcendental equation. Once the unknown constant $a$ has been determined, the corresponding maximum absolute error is obtained from the Eq. (3.2). A sequence of approximations for $a$ and the corresponding maximum absolute errors for $n=5,10$ and 12 are shown in Table 1 . As expected, this sequence converges to $a$ with the high convergence rate.

Table 1: Example 3.1. Constant $a$ and maximum absolute errors (MAEs).

\begin{tabular}{||c|cccc||}
\hline$n$ & 5 & 10 & 12 & Exact [39] \\
\hline$a$ & 0.3167048552978 & 0.316694366798 & 0.3166943676198 & 0.3166943676407 \\
$\mathrm{MAE}$ & $3.15 \times 10^{-9}$ & $4.88 \times 10^{-21}$ & $2.36 \times 10^{-26}$ & - \\
\hline
\end{tabular}


Example 3.2. The nonlinear singular boundary value problem

$$
\begin{aligned}
& y^{\prime \prime}+\frac{2}{x} y^{\prime}+\mathrm{e}^{-y}=0, \\
& y^{\prime}(0)=0, \quad 0.1 y(1)+y^{\prime}(1)=0
\end{aligned}
$$

is used in a heat conduction model of the human head [26].

In this case, the variational iteration algorithm II is

$$
y_{n+1}(x)=y_{0}(x)+\int_{0}^{x} \frac{s(s-x)}{x} \sum_{k=0}^{n} A_{k}(s) \mathrm{d} s,
$$

and the corresponding first four Adomian polynomials are

$$
\begin{aligned}
& A_{0}=\mathrm{e}^{-u_{0}}, \quad A_{1}=-\mathrm{e}^{-u_{0}} u_{1} \\
& A_{2}=\frac{1}{2} \mathrm{e}^{-u_{0}} u_{1}^{2}-\mathrm{e}^{-u_{0}} u_{2} \\
& A_{3}=-\frac{1}{6} \mathrm{e}^{-u_{0}} u_{1}^{3}+\mathrm{e}^{-u_{0}} u_{1} u_{2}-\mathrm{e}^{-u_{0}} u_{3} .
\end{aligned}
$$

Taking $y_{0}=a$ as the initial approximation, produces

$$
\begin{aligned}
& y_{1}(x)=a-\frac{\mathrm{e}^{-a}}{6} x^{2}, \\
& y_{2}(x)=a-\frac{\mathrm{e}^{-a}}{6} x^{2}-\frac{\mathrm{e}^{-2 a}}{120} x^{4}, \\
& y_{3}(x)=a-\frac{\mathrm{e}^{-a}}{6} x^{2}-\frac{\mathrm{e}^{-2 a}}{120} x^{4}-\frac{\mathrm{e}^{-3 a}}{1890} x^{6}, \\
& y_{4}(x)=a-\frac{\mathrm{e}^{-a}}{6} x^{2}-\frac{\mathrm{e}^{-2 a}}{120} x^{4}-\frac{\mathrm{e}^{-3 a}}{1890} x^{6}-\frac{61 \mathrm{e}^{-4 a}}{1632960} x^{8} .
\end{aligned}
$$

In this case, $M=K=1-\mathrm{cf}$. Ref. [17]. Consequently, the sequence $y_{n}(x)$ converges to the exact solution in the space $C_{[0,1]}$ and

$$
E_{n} \leq \frac{1}{(2 n+3) !}
$$

The approximations of $a$ and the corresponding maximum absolute errors are presented in Table 2. It shows the rapid convergence of the approximation sequence.

Table 2: Example 3.2. Constant $a$ and maximum absolute errors (MAEs).

\begin{tabular}{||c|cccc||}
\hline$n$ & 1 & 5 & 9 & 10 \\
\hline$a$ & 1.130289326974 & 1.147039006534 & 1.14703901933 & 1.14703901933 \\
MAE & $8.34 \times 10^{-3}$ & $1.61 \times 10^{-10}$ & $1.96 \times 10^{-20}$ & $3.87 \times 10^{-23}$ \\
\hline
\end{tabular}


Example 3.3. Consider the following nonlinear boundary value problem:

$$
\begin{aligned}
& y^{\prime \prime}+\mathrm{e}^{x+y}=1, \\
& y^{\prime}(0)=-1, \quad y(1)=-1
\end{aligned}
$$

The corresponding variational iteration algorithm II has the form

$$
y_{n+1}(x)=y_{0}(x)+\int_{0}^{x}(s-x) \sum_{k=0}^{n} A_{k}(s) \mathrm{d} s,
$$

and the first four Adomian polynomials are

$$
\begin{aligned}
& A_{0}=\mathrm{e}^{x+u_{0}}-1, \quad A_{1}=\mathrm{e}^{x+u_{0}} u_{1}, \\
& A_{2}=\frac{1}{2} \mathrm{e}^{x+u_{0}} u_{1}^{2}+\mathrm{e}^{x+u_{0}} u_{2}, \\
& A_{3}=\frac{1}{6} \mathrm{e}^{x+u_{0}} u_{1}^{3}+\mathrm{e}^{x+u_{0}} u_{1} u_{2}+\mathrm{e}^{x+u_{0}} u_{3} .
\end{aligned}
$$

Taking $y_{0}=a-x$ as the initial approximation, we obtain

$$
\begin{aligned}
y_{1}(x)= & a-x-\frac{\mathrm{e}^{a}-1}{2} x^{2}, \\
y_{2}(x)= & a-x-\frac{\mathrm{e}^{a}-1}{2} x^{2}+\frac{\mathrm{e}^{a}\left(\mathrm{e}^{a}-1\right)}{24} x^{4}, \\
y_{3}(x)= & a-x-\frac{\mathrm{e}^{a}-1}{2} x^{2}+\frac{\mathrm{e}^{a}\left(\mathrm{e}^{a}-1\right)}{24} x^{4}-\frac{3 \mathrm{e}^{a}-7 \mathrm{e}^{2 a}+4 \mathrm{e}^{3 a}}{720} x^{6}, \\
y_{4}(x)= & a-x-\frac{\mathrm{e}^{a}-1}{2} x^{2}+\frac{\mathrm{e}^{a}\left(\mathrm{e}^{a}-1\right)}{24} x^{4}-\frac{3 \mathrm{e}^{a}-7 \mathrm{e}^{2 a}+4 \mathrm{e}^{3 a}}{720} x^{6} \\
& -\frac{15 \mathrm{e}^{a}-63 \mathrm{e}^{2 a}+82 \mathrm{e}^{3 a}-34 \mathrm{e}^{4 a}}{40320} .
\end{aligned}
$$

Arguments analogous to those in Example 3.1 show that $M=\mathrm{e}^{a+1}-1, K=\mathrm{e}^{a+1}$ and

$$
E_{n} \leq \frac{\mathrm{e}^{n(a+1)}\left(\mathrm{e}^{a+1}-1\right)}{(2 n+2) !}
$$

Proceeding as before, the values of $a$ for $n=1,10$ and 20 are found to be the same as 0 . This leads to function $y(x)=-x$, which is the exact solution of the problem.

\section{Conclusion}

We apply a variational iteration method using Adomian polynomials to strongly nonlinear boundary value problems. This approach involves the calculation of symbolic integrals connected with variational iteration methods. Error estimates have been derived and the convergence of the method established. Illustrative examples demonstrate the efficiency of the algorithm. 


\section{Acknowledgments}

This work was supported by the Ministry of Science and Technology, Taiwan, R.O.C under Grant No. MOST 105-2221-E-269-003.

\section{A. Appendix}

Define a function $g(x)$ by

$$
g(x)=\int_{0}^{x} \lambda(s ; x) s^{k} \mathrm{~d} s, \quad k \geq 0,
$$

where $\lambda(s ; x)$ is the Lagrange multiplier (2.2) such that

$$
\begin{aligned}
& \lambda(s=x ; x)=0, \\
& \left.\frac{\partial \lambda(s ; x)}{\partial x}\right|_{s=x}=-1, \\
& \frac{\partial^{2} \lambda(s ; x)}{\partial x^{2}}+\frac{m}{x} \frac{\partial \lambda(s ; x)}{\partial x}=0 .
\end{aligned}
$$

Calculating the derivative of $g$ in $x$ and using Leibnitz formula yields

$$
\begin{aligned}
& g^{\prime}(x)=\int_{0}^{x} \frac{\partial \lambda(s ; x)}{\partial x} s^{k} \mathrm{~d} s, \\
& g^{\prime \prime}(x)=\int_{0}^{x} \frac{\partial^{2} \lambda(s ; x)}{\partial x^{2}} s^{k} \mathrm{~d} s-x^{k} .
\end{aligned}
$$

It follows that

$$
g^{\prime \prime}(x)+\frac{m}{x} g^{\prime}(x)+x^{k}=0
$$

or

$$
\frac{\mathrm{d}}{\mathrm{d} x}\left[x^{m} g^{\prime}(x)\right]=-x^{k+m} \text {. }
$$

Integrating the above expression twice and using the conditions $g(0)=g^{\prime}(0)=0$, one obtains

$$
g(x)=\int_{0}^{x} \lambda(s ; x) s^{k} \mathrm{~d} s=\frac{-1}{(k+m+1)(k+2)} x^{k+2} .
$$

\section{References}

[1] T.A. Abassy, M.A. El-Tawil, and H. El Zoheiry, Solving nonlinear partial differential equations using the modified variational iteration-Padé technique, J. Comput. Appl. Math. 207, 73-91 (2007).

[2] K. Abbaoui and Y. Cherruault, New ideas for proving convergence of decomposition method, Comput. Math. Appl. 29, 103-108 (1995). 
[3] S. Abbasbandy, A new application of He's variational iteration method for quadratic Riccati differential equation by using Adomian's polynomials, J. Comput. Appl. Math. 207, 59-63 (2007).

[4] M.A. Abdou, On the variational iteration method, Phys. Lett. A 366, 61-68 (2007).

[5] M. Abolhasani, S. Abbasbandy, and T. Allahviranloo, A new variational iteration method for a class of fractional convection-diffusion equations in large domains, Mathematics 5, 26 (2017).

[6] E.M. Abulwafa, M.A. Abdou, and A.A. Mahmoud, Nonlinear fluid flows in pipe-like domain problem using variational-iteration method, Chaos Solitons Fractals 32, 1384-1397 (2007).

[7] J.A. Adam and S.A. Maggelakis, Mathematical model of tumor growth IV: Effect of necrotic core, Math. Biosci. 97, 121-136 (1989).

[8] G. Adomian, Nonlinear stochastic differential equations, J. Math. Anal. Appl. 55, 443-452 (1976).

[9] G. Adomian and R. Rach, Inversion of nonlinear stochastic operator, J. Math. Anal. Appl. 91, 39-46 (1983).

[10] P.L. Chambre, On the solution of the Poisson-Boltzmann equation with application to the theory of thermal explosions, J. Comput. Phys. 20, 1795-1797 (1952).

[11] S.-H. Chang, Transient electroosmotic flow in cylindrical microcapillaries containing salt-free medium, Biomicrofluidics 3, 012802 (2009).

[12] S.-H. Chang, Electroosmotic flow in slit microchannel containing salt-free solution, Eur. J. Mech. B Fluids 29, 337-341 (2010).

[13] S.-H. Chang, A variational iteration method for solving Troesch's problem, J. Comput. Appl. Math. 234, 3043-3047 (2010).

[14] S.-H. Chang, An efficient method for solving Troesch's problem, Adv. Sci. Lett. 9, 920-924 (2012).

[15] S.-H. Chang, Electroosmotic flow in a dissimilarly charged slit microchannel containing salt-free solution, Eur. J. Mech. B/Fluids 34, 85-90 (2012).

[16] S.-H. Chang, Taylor series method for solving a class of nonlinear singular boundary value problems arising in applied science, Appl. Math. Comput. 235, 110-117 (2014).

[17] S.-H. Chang, Convergence of variational iteration method applied to two-point diffusion problems, Appl. Math. Model. 40, 6805-6810 (2016).

[18] S.-H. Chang and I.-L. Chang, A new algorithm for calculating one-dimensional differential transform of nonlinear functions, Appl. Math. Comput. 195, 799-808 (2008).

[19] M.M. Chawla and P.N. Shivkumar, On the existence of solutions of a class of singular two-point nonlinear boundary value problems, J. Comput. Appl. Math. 19, 379-388 (1987).

[20] W. Chen and Z. Lu, An algorithm for Adomian decomposition method, Appl. Math. Comput. 159, 221-235 (2004).

[21] H.W. Choi and J.G. Shin, Symbolic implementation of the algorithm for calculating Adomian polynomials, Appl. Math. Comput. 146, 257-271 (2003).

[22] J.S. Duan, Recurrence triangle for Adomian polynomials, Appl. Math. Comput. 216, 1235-1241 (2010).

[23] J.S. Duan, Convenient analytic recurrence algorithms for the Adomian polynomials, Appl. Math. Comput. 217, 6337-6348 (2011).

[24] S.A. El-Wakil and M.A. Abdou, New applications of variational iteration method using Adomian polynomials, Nonlinear Dyn. 52, 41-49 (2008).

[25] S.A. El-Wakil, E.M. Abulwafa, and M.A. Abdou, An improved variational iteration method for solving coupled KdV and Boussinesq-like B $(m, n)$ equations, Chaos Solitons Fractals 39, 12321237 (2009).

[26] U. Flesch, The distribution of heat sources in the human head: a theoretical consideration, J. Theor. Biol. 54, 285-287 (1975). 
[27] J.B. Garner and R. Shivaji, Diffusion problems with a mixed nonlinear boundary condition, J. Math. Anal. Appl. 148, 422-430 (1990).

[28] H. Ghaneai and M.M. Hosseini, Variational iteration method with an auxiliary parameter for solving wave-like and heat-like equations in large domains, Comput. Math. Appl. 69, 363-373 (2015).

[29] H. Ghaneai and M.M. Hosseini, Solving differential-algebraic equations through variational iteration method with an auxiliary parameter, Appl. Math. Model. 40, 3991-4001 (2016).

[30] J.H. He, A new approach to nonlinear partial differential equations, Commun. Nonlinear Sci. Numer. Simul. 2, 230-235 (1997).

[31] J.H. He, Variational iteration method for delay differential equations, Commun. Nonlinear Sci. Numer. Simul. 2, 235-236 (1997).

[32] J.H. He, Variational iteration method - a kind of nonlinear analytical technique: Some examples, Int. J. Nonlinear Mech. 34, 699-708 (1999).

[33] J.H. He, Variational iteration method for autonomous ordinary systems, Appl. Math. Comput. 114, 115-123 (2000).

[34] J.H. He, A short remark on fractional variational iteration method, Phys. Lett. A 375, 33623364 (2011).

[35] J.H. He, Notes on the optimal variational iteration method, Appl. Math. Lett. 25, 1579-1581 (2012).

[36] J.H. He, G.C. Wu, and F. Austin, The variational iteration method which should be followed, Nonlinear Sci. Lett. A 1, 1-30 (2010).

[37] J.H. He and X.H. Wu, Variational iteration method: New development and applications, Comput. Math. Appl. 54, 881-894 (2007).

[38] N. Herişanu and V. Marinca, A modified variational iteration method for strongly nonlinear problems, Nonlinear Sci. Lett. A 1, 183-192 (2010).

[39] M. Kumar and N. Singh, Modified Adomian decomposition method and computer implementation for solving singular boundary value problems arising in various physical problems, Comput. Chem. Eng. 52, 1750-1760 (2010).

[40] Z.B. Li, W.H. Zhu, and J.H. He, Exact solutions of time-fractional heat conduction equation by the fractional complex transform, Therm. Sci. 16, 335-338 (2012).

[41] H. Liu, A. Xiao, and L. Su, Convergence of variational iteration method for second-order delay differential equations, J. Appl. Math. 2013, 634670 (2013).

[42] D.L.S. McElwain, A re-examination of oxygen diffusion in a spherical cell with Michaelis-Menten nonlinear oxygen uptake kinetics, J. Theor. Biol. 71, 255-263 (1978).

[43] F.M. Murray and K.S. Miller, Existence Theorems for Ordinary Differential Equations, New York University Press, New York (1954).

[44] Z. Odibat and S. Momani, Numerical methods for solving nonlinear partial differential equations of fractional order, Appl. Math. Model. 32, 28-39 (2008).

[45] Z.M. Odibat, A study on the convergence of variational iteration method, Math. Comput. Model. 51, 1181-1192 (2010).

[46] A. Pourdarvish, A reliable symbolic implementation of algorithm for calculating Adomian polynomials, Appl. Math. Comput. 172, 545-550 (2006).

[47] R. Rach, A convenient computational form for the Adomian polynomials, J. Math. Anal. Appl. 102, 415-419 (1984).

[48] R. Rach, A new definition of the Adomian polynomials, Kybernetes 37, 910-955 (2008).

[49] A.S.V. Ravi Kanth and K. Aruna, He's variational iteration method for treating nonlinear singular boundary value problems, Comput. Math. Appl. 60, 821-829 (2010).

[50] G. Rezazadeh, H. Madinei, and R. Shabani, Study of parametric oscillation of an electrostati- 
cally actuated microbeam using variational iteration method, Appl. Math. Model. 36, 403-443 (2012).

[51] M.G. Sakar and H. Ergören, Alternative variational iteration method for solving the timefractional Fornberg-Whitham equation, Appl. Math. Model. 39, 3972-3979 (2015).

[52] D.K. Salkuyeh, Convergence of the variational iteration method for solving linear systems of ODEs with constant coefficients, Comput. Math. Appl. 56, 2027-2033 (2008).

[53] D.K. Salkuyeh and A. Tavakoli, Interpolated variational iteration method for initial value problems, Appl. Math. Model. 40, 3979-3990 (2016).

[54] N.H. Sweilam, Harmonic wave generation in nonlinear thermoelasticity by variational iteration method and Adomian's method, J. Comput. Appl. Math. 207, 64-72 (2007).

[55] N.H. Sweilam and M.M. Khader, Variational iteration method for one dimensional nonlinear thermoelasticity, Chaos Solitons Fractals 32, 145-149 (2007).

[56] M. Turkyilmazoglu, An optimal variational iteration method, Appl. Math. Lett. 24, 762-765 (2011).

[57] A.M. Wazwaz, A new algorithm for calculating Adomian polynomials for nonlinear operators, Appl. Math. Comput. 111, 53-69 (2000).

[58] A.M. Wazwaz, The variational iteration method for solving nonlinear singular boundary value problems arising in various physical models, Commun. Nonlinear Sci. Numer. Simul. 16, 38813886 (2011).

[59] G. Wu and E.W.M. Lee, Fractional variational iteration method and its application, Phys. Lett. A 374, 2506-2509 (2010).

[60] G.C. Wu, Laplace transform overcoming principles drawbacks in application of the variational iteration method to fractional heat equations, Therm. Sci. 16, 1257-1261 (2012).

[61] G.C. Wu and D. Baleanu, Variational iteration method for the Burgers' flow with fractional derivatives-New Lagrange multipliers, Appl. Math. Model. 37, 6183-6190 (2013). 